\title{
A GEOMETRIC METHOD IN DIFFERENTIAL TOPOLOGY
}

\author{
BY ANDREW H. WALLACE
}

Introduction. My aim here is to explain in an informal way a technique which has proved itself useful in dealing with certain problems in differential topology. The title refers to the fact that the method relies principally on the geometric construction of spherical modifications and certain associated constructions. The general plan of this exposition is, firstly, to define the notion of spherical modification, secondly, to describe some operations which can be performed on these modifications, and thirdly, to give some applications. The article concludes with some remarks on related work. A more formal and detailed presentation of the material treated here appears in $[3 ; 4]$.

1. Definition. Let $M_{1}$ be a differentiable manifold of dimension $n$ and let $S^{r}$ be an $r$-sphere embedded in $M_{1}$. If $S^{r}$ has a neighborhood in $M_{1}$ of the form $S^{r} \times E^{n-r}$, where $E^{n-r}$ is an $(n-r)$-cell, then $S^{r}$ is said to be directly embedded in $M_{1}$. If, in this case, $S^{r} \times E^{n-r}$ is removed from $M_{1}$, what is left is a manifold having a boundary of the form $S^{r} \times S^{n-r-1}$. The latter set is, however, also the boundary of $E^{r+1} \times S^{n-r-1}$. Thus, forming the union

$$
\left\{M_{1}-\left(S^{r} \times E^{n-r}\right)\right\} \cup\left(E^{r+1} \times S^{n-r-1}\right)
$$

with the appropriate identification of boundaries, a new manifold $M_{2}$ is obtained, which can also be made differentiable by a suitable smoothing operation. The manifold $M_{2}$ is said to be obtained from $M_{1}$ by a spherical modification.

A familiar example of this process is obtained by taking $M_{1}$ to be the hyperboloid of two sheets $x^{2}-y^{2}-z^{2}=1$ and $M_{2}$ to be the hyperboloid of one sheet $x^{2}-y^{2}-z^{2}=-1$ in Euclidean 3-space. $S^{r}$ is to be taken as the 0 -sphere consisting of the union of the two points $(-1,0,0)$ and $(1,0,0)$. In this case the set removed is a pair of discs and the set inserted is a circular cylinder.

More generally, $M_{i}$ could be taken as the quadric hypersurface

$$
\sum_{i=1}^{r+1} x_{i}^{2}-\sum_{i=r+2}^{n+1} x_{i}^{2}=1
$$

in $(n+1)$-space, and $M_{2}$ as the hypersurface whose equation is obtained from the above by changing the right hand side to -1 . In this

An address delivered before the Chicago meeting of the Society on April 13, 1962, by invitation of the Committee to Select Hour Speakers for Western Sectional Meetings; received by the editors June 15, 1962. 
example it is easily seen that one can make a continuous transition from $M_{1}$ to $M_{2}$, namely by passing through the family of quadrics

$$
\sum_{i=1}^{r+1} x_{i}^{2}-\sum_{i=r+2}^{n+1} x_{i}^{2}=t,
$$

allowing $t$ to vary from 1 to -1 . All the quadrics in this family are nonsingular except when $t=0$, in which case the hypersurface has a nondegenerate quadratic singularity. Also, still referring to this example, the family of orthogonal trajectories $F$ to the family (1) can be constructed. The family of curves $F$ will have one singular point, namely the origin. All the members of $F$ meeting the $r$-sphere $S^{r}$ in $M_{1}$ obtained from (1) by setting $t=1$ and $x_{i}=0$ for $i \geqq r+2$ or meeting the $(n-r-1)$-sphere $S^{n-r-1}$ in $M_{2}$ obtained by setting $t=-1$ and $x_{i}=0$ for $i \leqq r+1$ end at the origin. And all the members of $F$ meeting neighborhoods of these spheres in $M_{1}$ and $M_{2}$ fill out an $(n+1)$ cell, while the union of the remaining members of $F$ makes up the product $\left(M_{1}-\left(S^{r} \times E^{n-r}\right)\right) \times I$.

The description just given applies not only to this example, but to any transformation of a manifold $M_{1}$ to $M_{2}$ by a spherical modification. This follows from the fact that the whole operation of a spherical modification, as defined earlier in this section, depends only on a neighborhood of the corresponding $r$-sphere $S^{r}$. Thus also in the general case $M_{1}$ and $M_{2}$ form the boundary of a manifold $M$, which will be called the trace of the modification, and there is a family $F$ of curves in $M$, one curve passing through each point with the exception of one singular point. These curves can be parametrized by a parameter $t$ so that $M_{1}$ and $M_{2}$ are the sections $t=0$ and $t=1$, respectively, and so that all the sections $t=$ constant are nonsingular with one exception. This exceptional section will have one nondegenerate quadratic singularity $P$, namely the singularity of the family $F$. In the notation used earlier in this section, it will be noted that the members of the family $F$ beginning on $S^{r}$ all end at $P$, and those ending at a certain $(n-r-1)$-sphere $S^{n-r-1}$ in $M_{2}$ all begin at $P$. For this reason it is convenient to think of the modification as shrinking $S^{r}$ and introducing $S^{n-r-1}$.

More generally now, suppose that $M_{2}$ is reached from $M_{1}$ by performing some finite number of spherical modifications. Their traces can be put together to form a manifold $M$ (to be called the trace of the set of modifications) with boundary $M_{1} \cup M_{2}$. Thus $M_{1}$ and $M_{2}$ are cobounding manifolds. Also the families of curves corresponding as described above to the modifications can be put together to form one family in $M$, one curve passing through each point with a finite number of exceptions, one for each modification. 
The converse of the result just stated is also true. Namely, if $M_{1}$ and $M_{2}$ are cobounding manifolds then there is a finite sequence of spherical modifications leading from $M_{1}$ to $M_{2}$. This can be proved by making a suitable embedding of the manifold $M$ whose boundary is $M_{1} \cup M_{2}$ in $N$-space so that $M_{1}$ and $M_{2}$ are the sections $x_{N}=0$ and $x_{N}=1$, respectively, while the sections of $M$ by $x_{N}=$ constant are all nonsingular with a finite number of exceptions having nondegenerate quadratic singularities. Each singular section then corresponds to one modification.

2. Rearrangement of modifications. Some operations on modifications are now to be described. The first, which will be considered in this section, is the operation of switching the order of performing two modifications, under suitable conditions. Referring to Figure 1 for

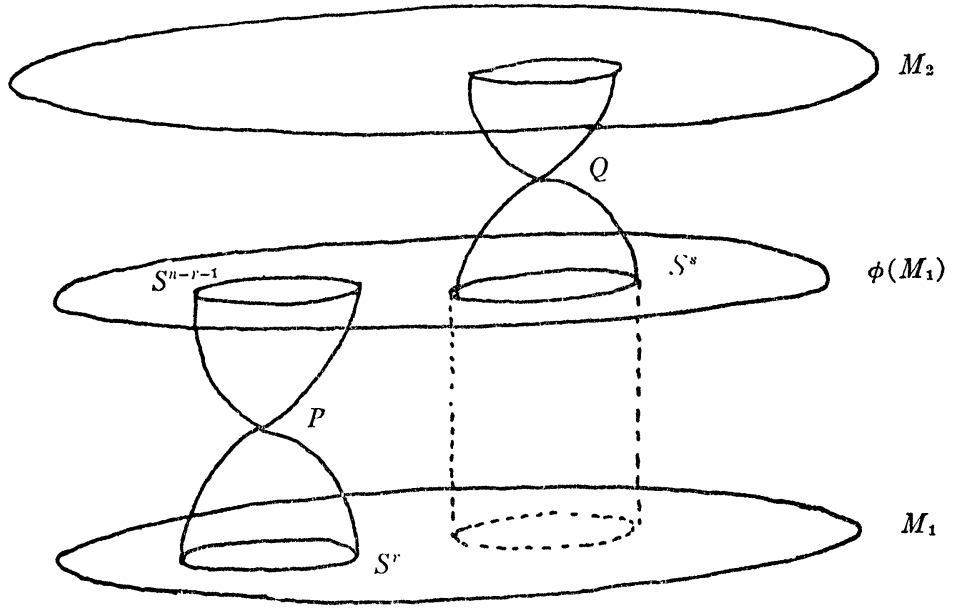

FIGURE 1.

guidance, let $M_{1}$ be transformed to $M_{2}$ by two modifications $\phi, \psi$, the combined trace of these modifications being $M . \phi$ shrinks the sphere $S^{r}$ to the singular point $P$ of the curve family $F$ associated with the modifications, and this modification introduces $S^{n-r-1}$. Let $\psi$, operating on $\phi\left(M_{1}\right)$, shrink the sphere $S^{s}$ to the singular point $Q$ of the family $F$ and introduce $S^{n-s-1}$. Assume that $S^{s} \cap S^{n-r-1}=\varnothing$. Then $S^{8}$ can be pulled back along the curves $F$ into $M_{1}$. Thus the two modifications $\phi$ and $\psi$ can both be thought of as operating on $M_{1}$. In the present arrangement, the modification $\phi$ is performed first, and so, as the parameter on the family $F$ increases from 0 to 1 , the singular point $P$ is reached before $Q$. However the parameter can be changed 
so that it increases faster on the curves of $F$ starting at points near $S^{r}$. The result will be that, as one proceeds along the curves $F$, using the new parameter, the singular point $Q$ is reached before $P$. Hence the modification $\psi$ is performed before $\phi$. That is to say, the order of carrying out the pair of modifications $\phi$ and $\psi$ has been reversed.

The condition for the above reversal in the order of $\phi$ and $\psi$ is that $S^{r} \cap S^{n-r-1}=\varnothing$. This can always be guaranteed if $s \leqq r$. For in this case $S^{s}$ can be made disjoint from $S^{n-r-1}$ by a small isotopic displacement, and it is clear that the result of a modification is unaffected by such a displacement of the sphere to be shrunk.

The result just obtained can be applied systematically to any finite sequence of modifications leading from $M_{1}$ to $M_{2}$. When this is done, it can be arranged that modifications shrinking $s$-spheres are performed before those shrinking $r$-spheres whenever $s<r$. And of course modifications shrinking spheres of the same dimension can be commuted freely among themselves.

3. Addition of modifications. The title of this section is a convenient but somewhat elliptical form of description, since the things which are actually to be added are elements of homology or homotopy groups.

(a)

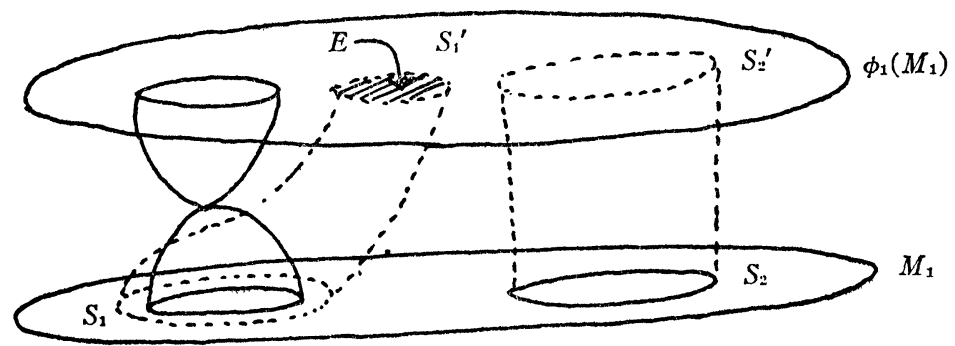

(b)

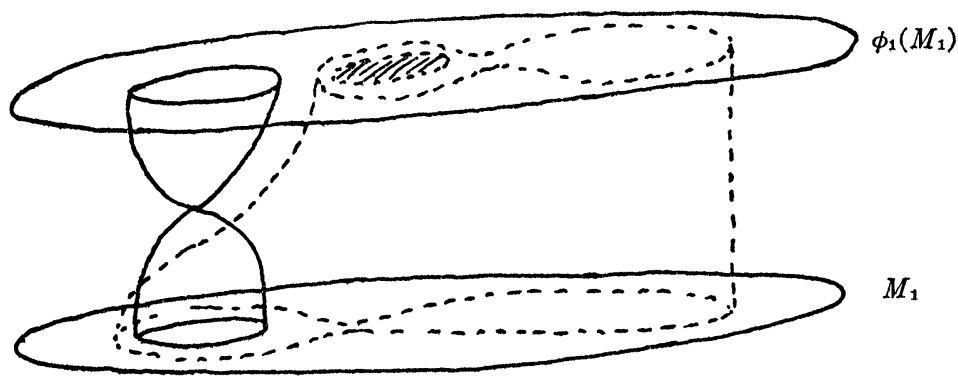

FigURE 2. (b) shows $S_{2}^{\prime}$ deformed across $E$, then pulled back into $M_{1}$. 
Let $\phi_{1}$ and $\phi_{2}$ be modifications shrinking $r$-spheres $S_{1}$, respectively $S_{2}$ in $M_{1}$, assumed disjoint, and let $S_{1}, S_{2}$ carry elements $\alpha_{1}, \alpha_{2}$, respectively, of $H_{r}\left(M_{1}\right)$. Suppose that the modification $\phi_{1}$ is done first. Then the curve family $F$ associated with $\phi_{1}$ can be used to pull a displaced copy of $S_{1}$ up into $\phi_{1}\left(M_{1}\right)$, giving a sphere $S_{1}^{\prime}$ in that manifold. $S_{1}^{\prime}$ will, however, bound a cell $E$ in $\phi_{1}\left(M_{1}\right) . S_{2}$, being disjoint from $S_{1}$, is carried into $\phi_{1}\left(M_{1}\right)$ by the curve family $F$, giving a sphere $S_{2}^{\prime}$ in $\phi_{1}\left(M_{1}\right) . \phi_{2}$ is now to be performed on $\phi_{1}\left(M_{1}\right)$ by shrinking the sphere $S_{2}^{\prime}$. But, as already remarked, the result of a modification is unaffected by an isotopic deformation of the sphere to be shrunk. In particular, $\phi_{2}$ gives the same result if $S_{2}^{\prime}$ is first deformed across the cell $E$. But now, if the displaced $S_{2}^{\prime}$ is pulled back into $M$ by means of the curve family $F$, a sphere is obtained which carries $\alpha_{2} \pm \alpha_{1}$ instead of $\alpha_{2}$, the sign depending on the way in which the deformation was made.

Hence the result of the pair of modifications $\phi_{1}, \phi_{2}$ is the same as that of a pair of modifications $\psi_{1}, \psi_{2}$ shrinking spheres carrying $\alpha_{1}$, $\alpha_{2} \pm \alpha_{1}$. Also the trace of the pair $\psi_{1}, \psi_{2}$ is the same as that of $\phi_{1}, \phi_{2}$.

Repeated application of this result shows that the pair of modifications $\phi_{1}, \phi_{2}$, shrinking spheres carrying $\alpha_{1}, \alpha_{2}$ in $H_{r}\left(M_{1}\right)$, gives the same result (and has the same trace) as a pair of modifications shrinking spheres carrying $\alpha_{1}$ and $\alpha_{2}+k \alpha_{1}$, where $k$ is any integer.

Again this result may be applied repeatedly to a set of modifications $\phi_{1}, \phi_{2}, \cdots, \phi_{h}$ shrinking disjoint $r$-spheres carrying $\alpha_{1}, \alpha_{2}, \cdots$, $\alpha_{h}$ in $H_{r}\left(M_{1}\right)$. These modifications will then give the same result and have the same trace as a set of modifications $\psi_{1}, \psi_{2}, \cdots, \psi_{h}$ shrinking spheres carrying $\beta_{1}, \beta_{2}, \cdots, \beta_{h}$ in $H_{r}\left(M_{1}\right)$, where the $\beta_{i}$ are obtained from the $\alpha_{j}$ by a unimodular linear transformation, for any such transformation can be expressed as a product of transformations of the type $\left(\alpha_{1}, \alpha_{2}\right) \rightarrow\left(\alpha_{1}, \alpha_{2}+k \alpha_{1}\right)$.

The disjointness of the spheres involved in this result will usually arise automatically in applications, the most important cases being where $2 r<\operatorname{dim} M_{1}$. The result is stated above in terms of homology, but a similar statement can be made in terms of homotopy groups, with suitable adjustments relative to the base point.

4. Complementary modifications and cancellation. Suppose that a modification $\phi$ on $M_{1}$ shrinks a sphere $S^{r}$ which is the boundary of an $(r+1)$-cell $E^{r+1}$. The effect of the modification is to close up $E^{r+1}$ to form an $(r+1)$-sphere $S^{r+1}$ in $\phi\left(M_{1}\right)$. Now suppose that this sphere $S^{r+1}$ is directly embedded and let $\psi$ be a modification shrinking it. $\psi$ will be said to be complementary to $\phi$.

When two modifications are related in this way, it can be shown 
that the final result $\psi \phi\left(M_{1}\right)$ is $M_{1}$ itself and that the trace of the pair $\phi, \psi$ is $M_{1} \times I$.

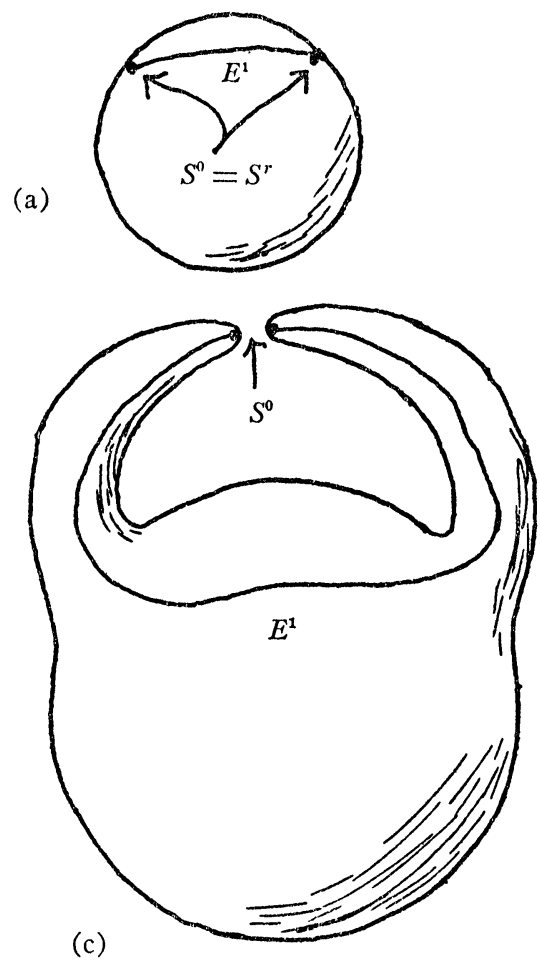

(b)
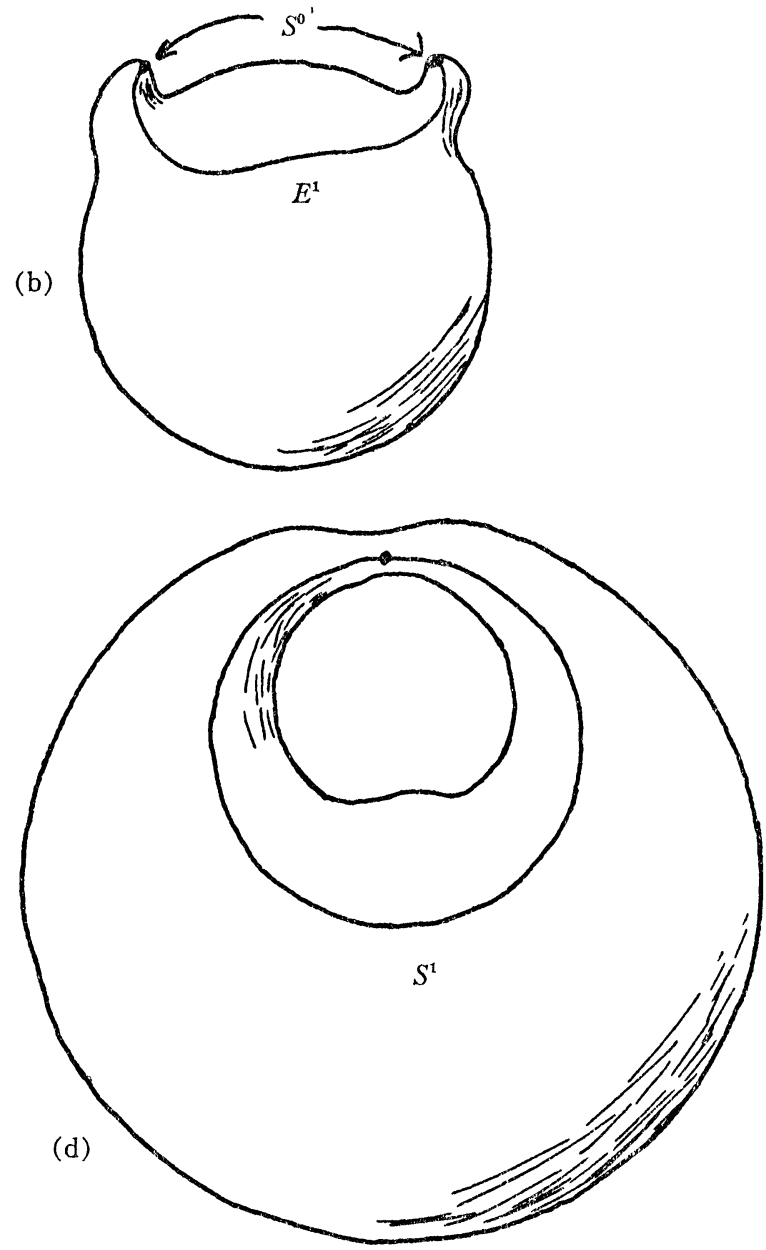

FIGURE 3. (a) $S^{r}=S^{0}$ is marked on $S^{2}$; here $t=0$.

(b) $t$ increases.

(c) $t$ increases further, and points forming $S^{0}$ are about to coalesce.

(d) Modification $\phi$ is completed. Surface is now a torus, and stages (a), (b), (c) are supposed to be inside the solid which it bounds.

To illustrate this (Figure 3) take $M_{1}=S^{2}$ and consider a modification $\phi$ shrinking a 0 -sphere to give a torus for $\phi\left(M_{1}\right)$. The trace of $\phi$ is a solid bounded by a sphere and a torus, that is to say, a solid torus with a spherical hole in it. If the family $F$ of curves correspond- 
ing to $\phi$ is parametrized by $t$, the set $t=0$ is the inner spherical boundary. As $t$ increases from zero the set $t=$ constant will be a sphere increasing in size, and growing horns around the points forming the 0 sphere to be shrunk by $\phi$. As $t$ increases these horns meet and join so that a torus is formed. The join of the two points forming $S^{r}$ is a 1-cell which $\phi$ closes up to form a circle $S^{1}$ on the outer surface of the solid torus. A second modification $\psi$ is to shrink $S^{1}$. Reasoning as above, it is clear that the trace of $\psi$ will be a solid having the torus as its inner boundary and a sphere as its outer boundary. Then the traces of $\phi$ and $\psi$ put together will form a solid sphere with a spherical hole in it, that is to say $S^{2} \times I$.

5. Applications. Suppose first that $M_{1}$ and $M_{2}$ form the boundary of the manifold $M$, and that the corresponding set of modifications leading from $M_{1}$ to $M_{2}$ consists of one, $\phi$, shrinking an $(r-1)$-sphere $S^{r-1}$ and a number of modifications shrinking $r$-spheres. The results of $\$ 2$ imply that $\phi$ can be assumed to be done first. Assume also that $\pi_{r}\left(M, M_{1}\right)=0$. This implies in particular that the $r$-cell $E_{1}$ bounded in $M$ by $S^{r-1}$ can be pulled back into $M_{1}$ giving a cell $E_{2}$ with boundary $S^{r-1}$ (cf. Figure 4). Then the modification $\phi$ shrinks $S^{r-1}$ and so closes up $E_{2}$ to form a sphere $S^{r}$. This is homotopic in $M$ to $E_{1} \cup E_{2}$ and so bounds a cell in $M$. Now there is a deformation retraction, using the curve family $F$, of $M$ onto the union of $\phi\left(M_{1}\right)$ with a number of cells, namely an $(n-r)$-cell bounded by the sphere introduced by $\phi$ and $(r+1)$-cells bounded by the spheres to be shrunk by the other modifications. Assuming here that $2 r<\operatorname{dim} M_{1}, S^{r}$ will thus carry an element of $\pi_{r}\left(\phi\left(M_{1}\right)\right)$ which is a linear combination of elements carried by $r$-spheres to be shrunk by modifications leading to $M_{2}$. Provided that $\operatorname{dim} M_{1} \geqq 5$, the result of $\$ 3$ can be applied to rearrange these modifications so that one of them actually shrinks $S^{r}$. This modification will be complementary to $\phi$ and so will cancel it out.

More generally the above cancellation can be carried out systematically for any $M_{1}$ and $M_{2}$ forming the boundary of a manifold $M$ and satisfying the conditions

$$
\begin{aligned}
\operatorname{dim} M_{1}=\operatorname{dim} M_{2} & =2 m \text { or } 2 m-1, \quad m \geqq 3, \\
\pi_{i}\left(M, M_{1}\right) & =0, \quad i \leqq m-1 .
\end{aligned}
$$

It will then follow that the corresponding modifications can be rearranged so that all those shrinking spheres of dimension less than $m-1$ cancel out.

If also $\pi_{i}\left(M, M_{2}\right)=0, i \leqq m-1$, the same reasoning can be carried out starting from $M_{2}$ and the result will be that all modifications will 
(a)

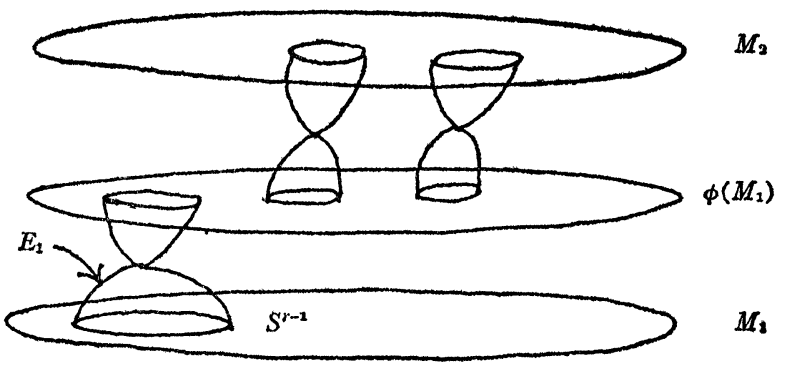

(b)

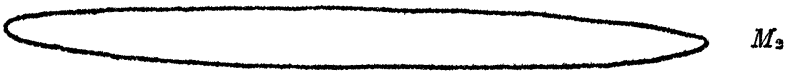

b)

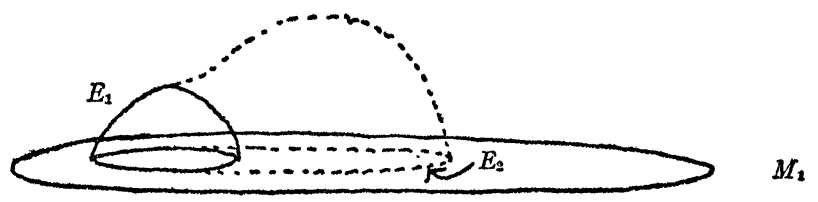

(c)
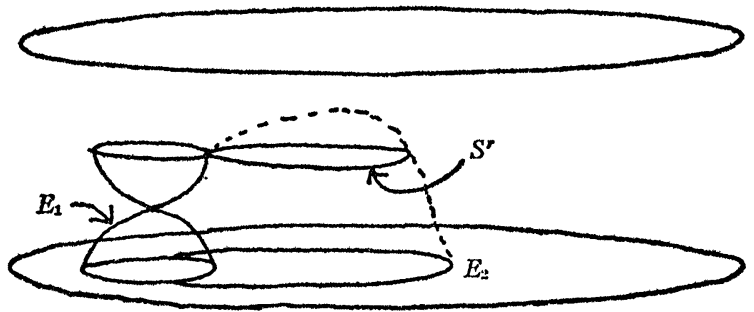

(d)

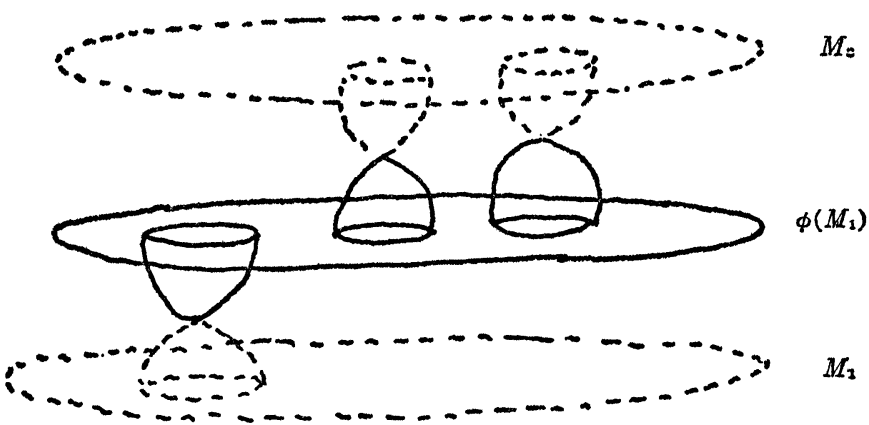

FIGURE 4 . (b) shows $E_{1}$ pulled back into $M_{1}$; (c) shows $E_{2}$ closed by $\phi$ to form $S^{r}$ bounding a cell in $M$; (d) shows $M$ retracted on $\phi\left(M_{1}\right)$ with one $(n-r)$-cell and several $(r+1)$-cells attached. 
cancel out except one or two types. Indeed it can be shown that if, in addition, $M_{1}$ and $M_{2}$ are simply connected and $\pi_{m}\left(M, M_{1}\right)$ $=\pi_{m}\left(M, M_{2}\right)=0$, then, using a refinement of the above methods, all the modifications cancel out. Thus under these conditions $M_{1}=M_{2}$ and $M=M_{1} \times I$.

In particular let $M$ be a simply connected homology sphere of dimension $n+1$. Remove two $(n+1)$-cells from $M$. The remainder $M^{\prime}$ has boundary $M_{1} \cup M_{2}$, where the $M_{i}$ are $n$-spheres, and $\pi_{i}\left(M^{\prime}, M_{j}\right)$ $=0$ for $j=1,2$ and $i \leqq n$. The above result implies that all the corresponding modifications leading from $M_{1}$ to $M_{2}$ cancel out, and so $M^{\prime}$ is $M_{1} \times I=S^{n} \times I$, for $n \geqq 5$. Hence $M=S^{n+1}$. This solves the Poincaré problem for dimensions greater than 5 .

6. Remarks. The operation called here a spherical modification appears elsewhere under various names, for example surgery, and $\chi$-construction $(c f .[1])$. One of the original applications [1] was to the problem of killing the homotopy groups of a manifold. The idea is to apply modifications shrinking spheres carrying generators of homotopy groups, when these spheres are directly embedded. A systematic scheme for such a killing process is described in [4] and in the forthcoming papers [5].

The situation described here is closely related to the theory of critical points of functions on a manifold. In the present notation if the curve family $F$ is parametrized by $t$, then the singular points of this family, corresponding to modifications leading from $M_{1}$ to $M_{2}$, are critical points of the function $t$ on $M$. The rearrangement of a sequence of modifications so that spheres of lower dimension are shrunk first corresponds to Smale's construction of a "nice function" on $M[2]$. And in fact Smale's approach to the generalized Poincaré problem is closely related to the above treatment, the manipulation of modifications being replaced by the construction of functions with suitable properties.

The problem which first led to my interest in this subject was that of giving some sort of geometrical account of the relation of cobordism or cobounding between manifolds. The homotopy killing process mentioned above can be applied to any manifold to give a cobounding manifold with a specially simple homotopy structure. If this is assumed done in advance to all manifolds, then, when two manifolds belong to the same cobordism class, they are related by modifications of only one or two types [4]. In forthcoming papers [5] this will be further strengthened. However, some essential refinement of method seems necessary before the ultimate ideal is attained, namely of giving 
in geometrical terms canonical representatives for the cobordism classes along with a geometrical algorithm for reducing a given manifold to the appropriate form.

\section{REFERENCES}

1. J. Milnor, A procedure for killing homotopy groups of differentiable manifolds, Differential Geometry, Proc. Sympos. Pure Math. Vol. 3, pp. 39-55, Amer. Math. Soc. Providence, R. I., 1961.

2. S. Smale, Poincaré conjecture in higher dimensions, Bull. Amer. Math. Soc. 66 (1960), 373-375.

3. A. H. Wallace, Modifications and cobounding manifolds, Canad. J. Math. 12 (1960), 503-528.

4. - Modifications and cobounding manifolds. II, J. Math. Mech. 10 (1961), 773-809.

5. - Modifications and cobounding manifolds. III, J. Math. Mech. 11 (1961); also IV. (to appear).

INDIANA UNIVERSITY 